\title{
Performance Management in West Sulawesi Regional Police
}

\author{
Vita Mayastinasari \\ Indonesia Police Science College, Jakarta Indonesia, $\bowtie$ mamayovi@gmail.com
}

\begin{abstract}
The performance of National Police cannot be separated from public assessment. Therefore, the consistency of positive performance is required, in other word, the national police should perform well every time. This research aims to identify public assessment of West Sulawesi Regional Police performance, analyze various programs of West Sulawesi Regional Police relating to performance and recommend formulation or strengthening performance management In West Sulawesi Regional Police. The problem in this research focused on public assessment of performance and management in West Sulawesi Regional Police. The approach used in this study is mix method research with survey methods and descriptive analysis. Respondent in this study involved 514 people. The research informant is National Police personnel. Data collection is conducted by questionnaire dissemination technique, Focus Group Discussion, observation and document collection. The Research instruments are questionnaires, interview guides, and check list documents. The results showed that public tendencies $(96.48 \%$ of respondents) assessed that National Police personnel in West Sulawesi performed well, with indications of timeliness, appropriateness and accuracy of police service results. In addition, West Sulawesi Regional Police personnels are also assessed to have adequate knowledge and skills, a willingness to help and a sense of empathy. The West Sulawesi police set up and implemented three programs to maintain and improve positive performance. The three programs, namely: Mi'oro Siola (Sitting together), Synergityof four pillars and Safari Shubuh performed together by Regional Police and district government.
\end{abstract}

Keywords: performance, management

\section{Introduction}

The National Police has duties as: protectors, protectors and public servants; Preserver of Community Security and Order (Kamtibmas) and Law Enforcement. Assessment of Indonesian National Police performance is inseparable from community assessment because police work is related to the community. Various surveys related to the assessment of National Police performance have been conducted by various external and internal institutions of National Police. One of them is research on level of public trust in National Police performance carried out continuously by National Police Research Centerlast four years, from 2015 to 2018. The results of the four-year study showed that public confidence in National Policeperformance increased. In a row, the level of public trustof Indonesian National performance Police in 2015 until 2018 amounting to: 65.92\%; 68.99\% 80.31\%, 82.32\% (Mayastinasari: 2018)

National Police is required to provide excellent service, so that Indonesian Police personnel must have the ability to anticipate and recognize community expectations, and exceed community expectations regarding police services. Conceptually, Solikin (2011) argued that achieving the goal of the realization of service quality is highly dependent on the suitability between service personnel, service standards and the people or communities served, illustrated in Figure 1. 


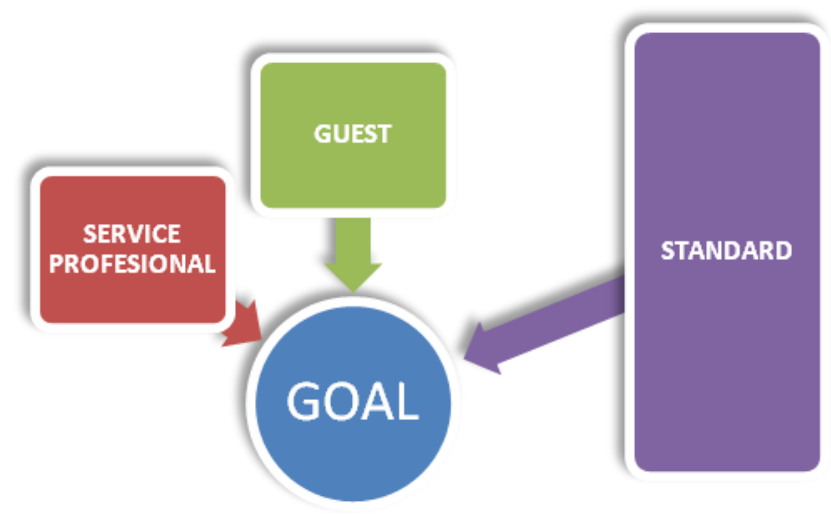

Figure 1 The Circle of Service

Resource Solikin (2011)

Police excellentservice is very linked with the police personnel professionalism. Therefore the qualified competence is needed. The needed competences are consists of cognitive ability, practical intelligence, emotional intelligence, physical abilities, social skills and attitude. Ability according to Colquitt, Jason, Lapine and Wesson(2013) refers to capabilities related to the acquisition and application of knowledge in problem solving. Ivancevich, Konopaske dan Matteson(2011, 74) stated: "Emotional intelligence refers to a person's ability to be self-aware of feelings, to manage emotions, to motivate oneself, to express empathy, and to handle relationships with others". Greenberg dan Baron $(2008,159)$ definedsocial skills as: "The capacity to interact effectively with others". Attitudewas defined by Schermerhorn, Hunt and Osborn $(2008,68)$ as: 'A predisposition to respond in a positive or negative way to someone or something in one's environmentt".

Indonesia National Police excellent service does not necessarily materialize without the application of a strategic and integrative management system with the involvement of all leaders and personnel. One part which needs to be observed to realize the excellent service of the police is the quality of internal services that have an impact on personnel satisfaction. Personnel satisfaction will encourage positive value of external services that have implications for community satisfaction and loyality. This is explained conceptually by Fandy Tjiptono, illustrated in Figure 2.

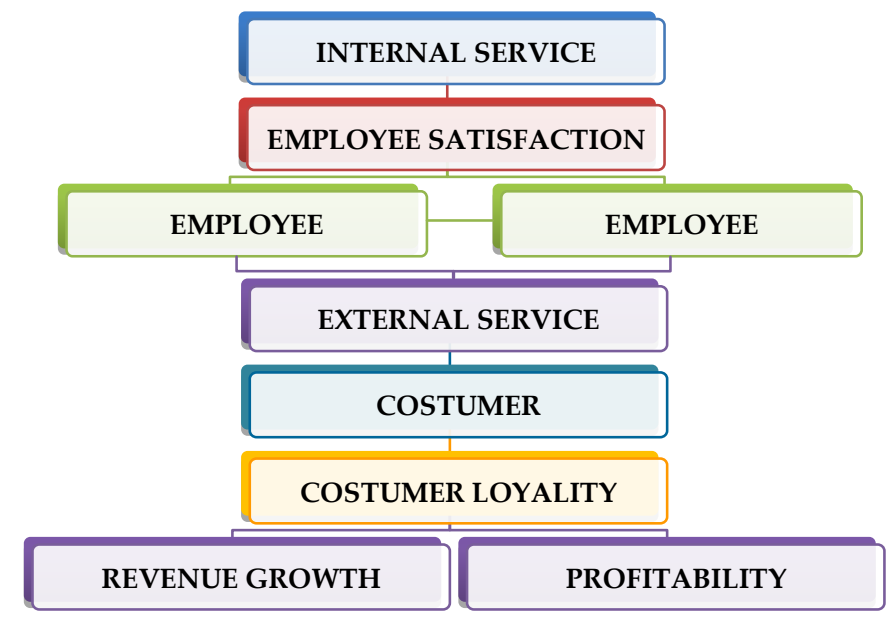

Figure 2 Service Profit Chain

Resource Fandy Tjiptono (1997)

Polri has thirty-three (33) Regional Police (Polda) at the time this research conducted. But currently there are thirty-four (34) Regional Police. The excellent service of the police can only be realized with 
the accuracy and consistency on implementation of strategic and integrative management system for all regional police. The West Sulawesi Regional Police is a relatively new regional police (33rd Regional Police), so the author is interested to conduct research related to performance in West Sulawesi Regional Police. This study aims to identify community assessments of of West Sulawesi Regional Policeperformance, analyze various West Sulawesi Regional Police programs regarding performance and recommend formulation or strengthening management performance.

The focus of the problems in this study are how is the community's assessment of National Police performance in West Sulawesi Regional Police?, How is the management of National Police performance management in West Sulawesi Regional Police?. Relating to this description, a study was conducted on: "Management of Performance Management at the West Sulawesi Regional Police".

\section{Methods}

This research was conducted with a mix method research approach. The method used is survey and descriptive analysis. The population in this study was the community and police personnel in West Sulawesi Regional Police. The respondents of this study are the community, as many as 514 people. The community of respondent is the community who knew and experienced police services at one, several or all functions in the five police functions studied. Research informants areNational Police personnels who have duties in Regional Police or at the Police Station. The informants of the National Police personnel at the Regional Police level are: a. Director of General Investigation; $b$. Special Detective Director; c. Director of Drug Narcotics; d. Directorate of Traffic; e. Director of Security Intelligence; f. Director of Community Development, g. Director Samapta Bhayangkara. While informants at resort police level including: a. Resort Police Chief (Kapolres); b. Deputy Chief of Resort Police (Wakapolres); c. Head of Operations Section (Head of Ops.); d. Head of Criminal Investigation Unit (Reskrim); e. Head of Traffic Unit ; f. Head of Security Intelligence Unit (Head of Intelligence Unit); g. Head of Narcotics Unit; h. Head of Samapta Bhayangkara Unit (Kasat Sabhara); i. Head of Community Development Unit (Kasat Binmas).

The study was conducted with the technique of distributing questionnaires, Focus Group Discussion (FGD), observation and document collection. The questionnaire was distributed to the community with the aim of identifying community perceptions of the performance of the National Police. The FGD was conducted to the community and police personnel to get an explanation and understanding of the performance of the National Police. Observation of police services is carried out by observation. Collection of documents is done to analyze rules related to police service standards. The instruments used in this study are: questionnaires, interview guides and document checl.

Data analysis was conducted by triangulation technique, analyzing the results of the questionnaire and FGD answers. The scope of the research is focused on performance appraisal on indicators: 1) direct evidence (tangibles); 2) Reliability; 3) Responsiveness; 4) Assurance; 5) Conformance. The research was also limited to the implementation of police duties in the five operational functions of the police, namely: Criminal Investigation, Traffic, Intelligence, Binmas and Sabhara.

\section{Result and Discussion}

Community assessment of National Police performance is an important thing that should be identified, because the assessment of National Police performance is not only determined by internal assessments with indicators of performance appraisal and predetermined work targets. This can be understood because National Police is a public institution that has responsibility to the community, so it is natural that community assessment is one of the important indicators of performance appraisal. Community research on National Police performance in five police functions, illustrated in Table 1 to Table 6.

The tendency of respondents, as much as $93.44 \%$, assessed both the performance of traffic functions. Specifically, $87.14 \%$ of respondents gave a tendency towards good judgment on direct evidence indicators; $92.79 \%$ reliability indicator, $96.52 \%$ responsiveness indicator, $95.19 \%$ guarantee 
indicator and $95.55 \%$ conformity indicator. An assessment of the performance of the National Police specifically on the function of traffic can be observed in Table 1

Table 1 Community Assessment of Traffic Function Performance in West Sulawesi Regional Police

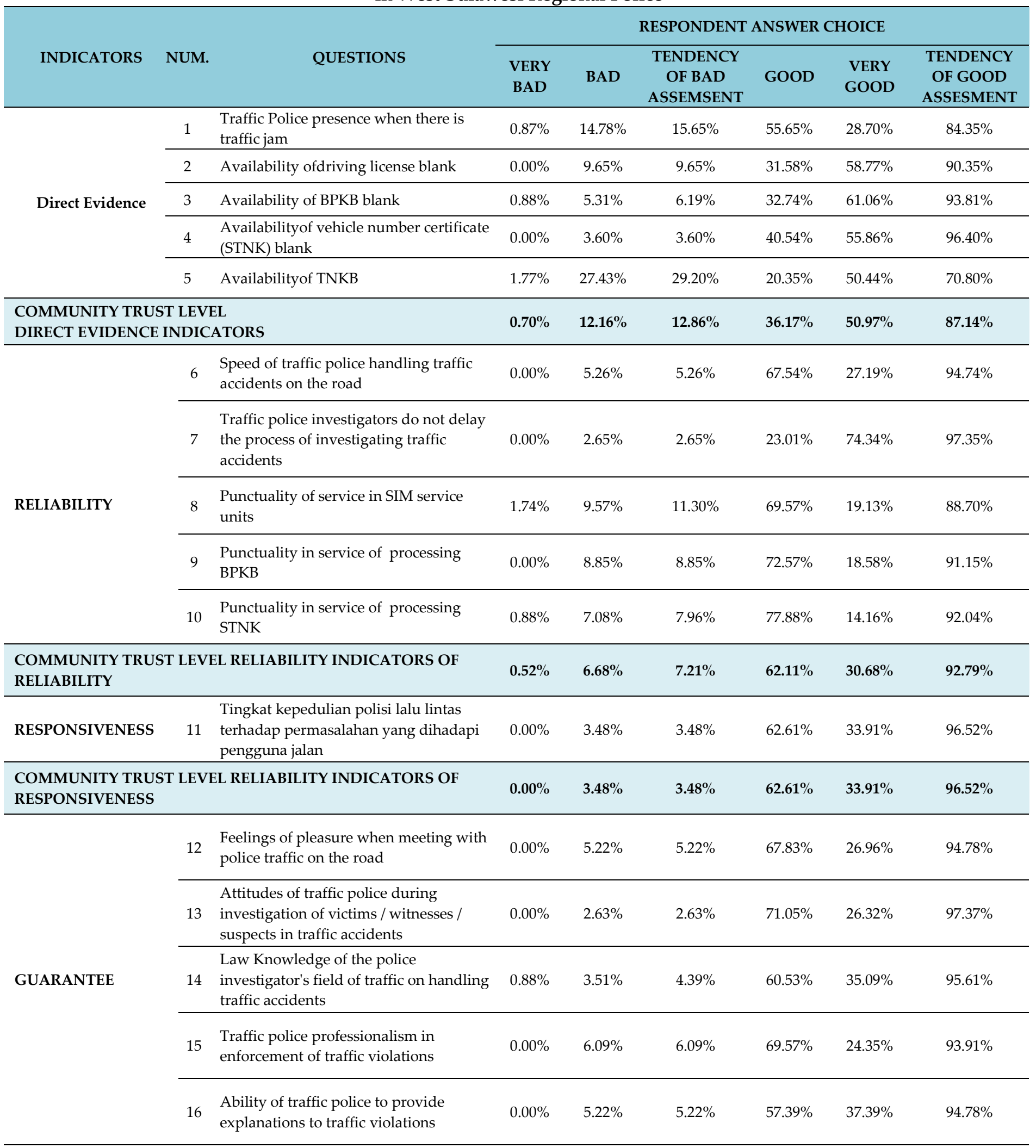


Table Cont...

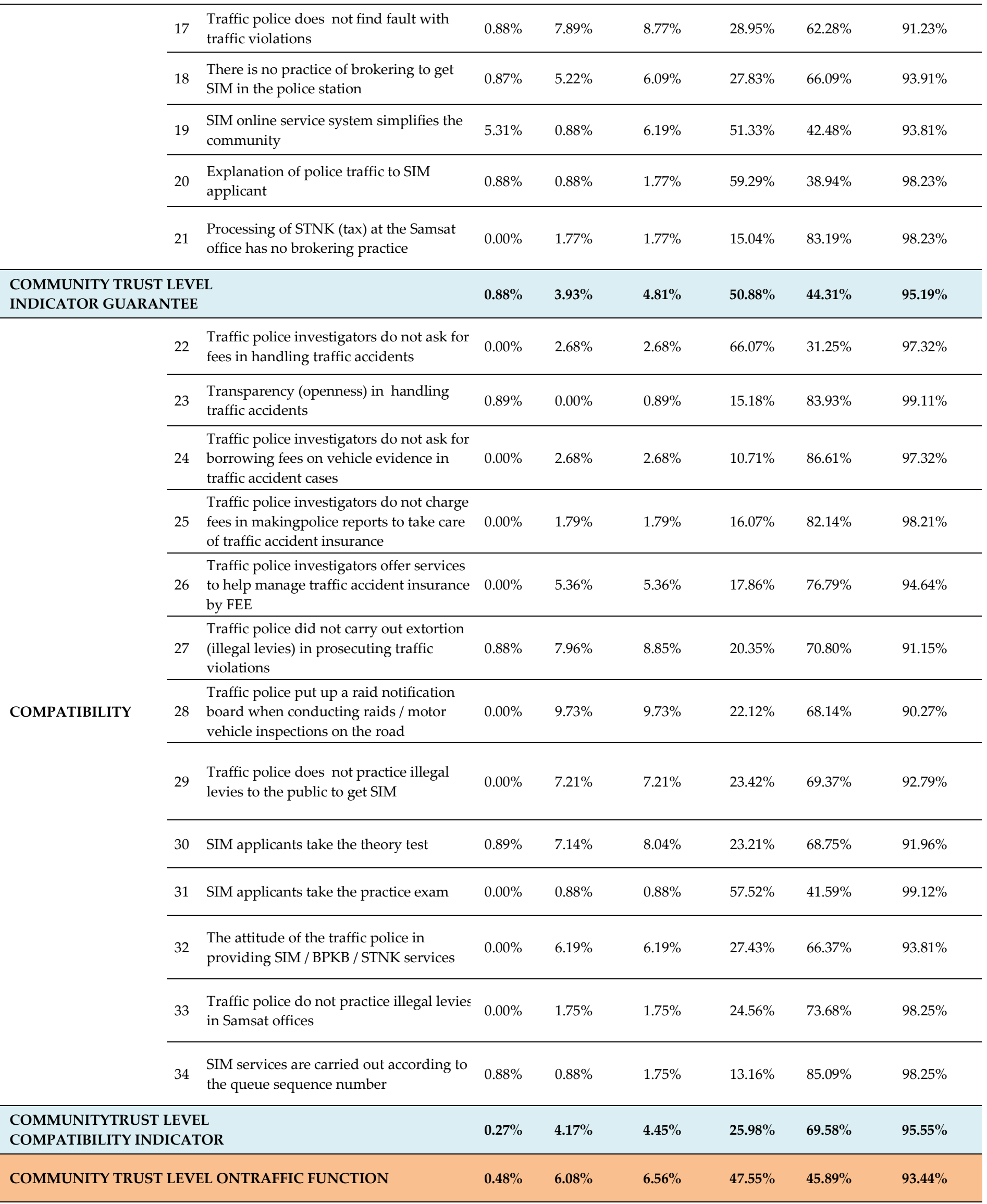

Source: Processed Data 
95.68\%respondents to the study stated that they had a good assessment of the services of the Reskrim function in West Sulawesi Regional Police. The indicator of direct evidence was well assessed by $99.08 \%$ of respondents. Reliability indicators were rated well by $93.64 \%$ of respondents. Indicators of responsiveness, guarantee indicators and consecutive indicators of conformity were given good evaluations by respondents: $97.30 \% ; 96.69 \%$; $91.71 \%$.

Table 2 Community Assessment of the of Criminal and Detective FunctionPerformance In West Sulawesi Regional Police

\begin{tabular}{|c|c|c|c|c|c|c|c|c|}
\hline \multirow[b]{2}{*}{ INDICATORS } & \multirow[b]{2}{*}{ NUM } & \multirow[b]{2}{*}{ QUESTIONS } & \multicolumn{6}{|c|}{ RESPONDENT ANSWER CHOICE } \\
\hline & & & $\begin{array}{l}\text { VERY } \\
\text { BAD }\end{array}$ & $\begin{array}{l}\text { VERY } \\
\text { BAD }\end{array}$ & $\begin{array}{l}\text { VERY } \\
\text { BAD }\end{array}$ & $\begin{array}{l}\text { VERY } \\
\text { BAD }\end{array}$ & $\begin{array}{l}\text { VERY } \\
\text { BAD }\end{array}$ & VERY BAD \\
\hline DIRECT EVIDENCE & 1 & $\begin{array}{l}\text { Giving Notification Letter of } \\
\text { Progress on Investigation } \\
\text { Progress (SP2HP) by Police } \\
\text { investigator to reporter }\end{array}$ & $0.00 \%$ & $0.92 \%$ & $0.92 \%$ & $25.69 \%$ & $73.39 \%$ & $99.08 \%$ \\
\hline \multicolumn{3}{|c|}{$\begin{array}{l}\text { COMMUNITY TRUST LEVEL } \\
\text { DIRECT EVIDENCE INDICATOR }\end{array}$} & $0.00 \%$ & $0.92 \%$ & $0.92 \%$ & $25.69 \%$ & $73.39 \%$ & $99.08 \%$ \\
\hline RELIABILITY & 2 & $\begin{array}{l}\text { Police investigators tend not } \\
\text { to delay the case handling } \\
\text { process }\end{array}$ & $0.91 \%$ & $5.45 \%$ & $6.36 \%$ & $56.36 \%$ & $37.27 \%$ & $93.64 \%$ \\
\hline \multicolumn{3}{|c|}{$\begin{array}{l}\text { COMMUNITY TRUST LEVEL } \\
\text { INDICATOR OF REALIBILITY }\end{array}$} & $0.91 \%$ & $5.45 \%$ & $6.36 \%$ & $56.36 \%$ & $37.27 \%$ & $93.64 \%$ \\
\hline RESPONSIVENESS & 3 & $\begin{array}{l}\text { The speed of case handling by } \\
\text { Polri investigators }\end{array}$ & $0.00 \%$ & $2.70 \%$ & $2.70 \%$ & $77.48 \%$ & $19.82 \%$ & $97.30 \%$ \\
\hline \multicolumn{3}{|c|}{$\begin{array}{l}\text { COMMUNITY TRUST LEVEL } \\
\text { INDICATOR OF RESPONSIVENESS }\end{array}$} & $0.00 \%$ & $2.70 \%$ & $2.70 \%$ & $77.48 \%$ & $19.82 \%$ & $97.30 \%$ \\
\hline \multirow{3}{*}{ GUARANTEE } & 4 & $\begin{array}{l}\text { The ability of the INP } \\
\text { investigator to provide an } \\
\text { explanation of cases / cases } \\
\text { handled }\end{array}$ & $0.00 \%$ & $0.90 \%$ & $0.90 \%$ & $54.05 \%$ & $45.05 \%$ & $99.10 \%$ \\
\hline & 5 & $\begin{array}{l}5 \text { Investigators do not } \\
\text { selectively handle corruption } \\
\text { cases }\end{array}$ & $2.06 \%$ & $5.15 \%$ & $7.22 \%$ & $6.19 \%$ & $86.60 \%$ & $92.78 \%$ \\
\hline & 6 & $\begin{array}{l}\text { The process of investigating } \\
\text { witnesses / suspects is not } \\
\text { accompanied by violence }\end{array}$ & $0.00 \%$ & $1.80 \%$ & $1.80 \%$ & $3.60 \%$ & $94.59 \%$ & $98.20 \%$ \\
\hline \multicolumn{3}{|c|}{$\begin{array}{l}\text { COMMUNITY TRUST LEVEL } \\
\text { INDICATOR OF GUARANTEE }\end{array}$} & $0.69 \%$ & $2.62 \%$ & $3.31 \%$ & $21.28 \%$ & $75.41 \%$ & $96.69 \%$ \\
\hline \multirow{4}{*}{$\begin{array}{l}\text { COMPITA } \\
\text { BILITY }\end{array}$} & 7 & $\begin{array}{l}\text { The attitude of the } \\
\text { investigator in investigation }\end{array}$ & $0.00 \%$ & $0.00 \%$ & $0.00 \%$ & $55.86 \%$ & $44.14 \%$ & $100.00 \%$ \\
\hline & 8 & $\begin{array}{l}\text { Police Investigators do not } \\
\text { carry out illegal levies in } \\
\text { handling case }\end{array}$ & $0.00 \%$ & $0.00 \%$ & $0.00 \%$ & $52.29 \%$ & $47.71 \%$ & $100.00 \%$ \\
\hline & 9 & $\begin{array}{l}\text { The National Police } \\
\text { Investigator explains the } \\
\text { rights and obligations to the } \\
\text { victim / witness / suspect at } \\
\text { the beginning of the } \\
\text { investigation }\end{array}$ & $0.00 \%$ & $2.75 \%$ & $2.75 \%$ & $11.01 \%$ & $86.24 \%$ & $97.25 \%$ \\
\hline & 10 & $\begin{array}{l}\text { Law enforcement is not } \\
\text { discriminatory (people are } \\
\text { treated unfairly) }\end{array}$ & $16.67 \%$ & $45.37 \%$ & $62.04 \%$ & $11.11 \%$ & $26.85 \%$ & $37.96 \%$ \\
\hline
\end{tabular}


Table Cont...

\begin{tabular}{|c|c|c|c|c|c|c|c|}
\hline 11 & $\begin{array}{l}\text { Settlement of cases at the } \\
\text { National Police is free from } \\
\text { KKN (corruption, collusion } \\
\text { and nepotism) }\end{array}$ & $0.00 \%$ & $0.00 \%$ & $0.00 \%$ & $24.07 \%$ & $75.93 \%$ & $100.00 \%$ \\
\hline 12 & $\begin{array}{l}\text { Police investigator is } \\
\text { professionalism in the } \\
\text { settlement of cases }\end{array}$ & $0.00 \%$ & $1.80 \%$ & $1.80 \%$ & $51.35 \%$ & $46.85 \%$ & $98.20 \%$ \\
\hline 13 & $\begin{array}{l}\text { The applicant is not asked for } \\
\text { compensation in submitting a } \\
\text { detention suspension }\end{array}$ & $0.00 \%$ & $1.83 \%$ & $1.83 \%$ & $7.34 \%$ & $90.83 \%$ & $98.17 \%$ \\
\hline 14 & $\begin{array}{l}\text { Investigators do not use their } \\
\text { authority to get compensation } \\
\text { by changing the status of } \\
\text { dealer and addicts / users / } \\
\text { victims of narcotics abuse }\end{array}$ & $1.02 \%$ & $2.04 \%$ & $3.06 \%$ & $7.14 \%$ & $89.80 \%$ & $96.94 \%$ \\
\hline 15 & $\begin{array}{l}\text { Police are not backing } \\
\text { (protecting) dealer and } \\
\text { addicts / users / victims of } \\
\text { narcotics abuse }\end{array}$ & $0.00 \%$ & $3.09 \%$ & $3.09 \%$ & $1.03 \%$ & $95.88 \%$ & $96.91 \%$ \\
\hline \multicolumn{2}{|c|}{$\begin{array}{l}\text { LEVEL } \\
\text { PATIBILTY }\end{array}$} & $1.97 \%$ & $6.32 \%$ & $8.29 \%$ & $24.58 \%$ & $67.13 \%$ & $91.71 \%$ \\
\hline \multicolumn{2}{|c|}{ LEVEL ON RESERSE FUNCTION } & $0.71 \%$ & $3.60 \%$ & $4.32 \%$ & $41.08 \%$ & $54.61 \%$ & $95.68 \%$ \\
\hline
\end{tabular}

Source: Processed Data

The function of the Intelkam in West Sulawesi Regional Police is considered to have good performance. This is indicated by the tendency of good assessment by $99.03 \%$ of respondents in this research. The informant who gave a good assessment tendency on the direct evidence indicator was $97.81 \%, 100 \%$ reliability indicator, $99.40 \%$ responsiveness indicator, $99.06 \%$ guarantee indicator and $98.87 \%$ compliance indicator. In detail the assessment results per indicator are illustrated in Table 3.

Table 3 Community Assessment of the Security Intelligence FunctionPerformance in West Sulawesi Regional Police

\begin{tabular}{|c|c|c|c|c|c|c|c|c|}
\hline \multirow[b]{2}{*}{ INDICATOR } & \multirow[b]{2}{*}{$\begin{array}{l}\text { NU } \\
\text { M }\end{array}$} & \multirow[b]{2}{*}{ QUESTIONS } & \multicolumn{6}{|c|}{ VERY BAD } \\
\hline & & & $\begin{array}{l}\text { VERY } \\
\text { BAD }\end{array}$ & BAD & $\begin{array}{c}\text { TENDENCY } \\
\text { OFBAD } \\
\text { ASSEMSENT }\end{array}$ & GOOD & $\begin{array}{l}\text { VERY } \\
\text { GOOD }\end{array}$ & $\begin{array}{c}\text { TENDENCY } \\
\text { OFGOOD } \\
\text { ASSESMENT }\end{array}$ \\
\hline \multirow{2}{*}{$\begin{array}{l}\text { DIRECT } \\
\text { EVIDENCE }\end{array}$} & 1 & $\begin{array}{l}\text { Availability of fingerprinting devices } \\
\text { at SKCK service points }\end{array}$ & $1.09 \%$ & $1.09 \%$ & $2.17 \%$ & $7.61 \%$ & $90.22 \%$ & $97.83 \%$ \\
\hline & 2 & $\begin{array}{l}\text { Availability of fingerprint blanks (AK } \\
\text { 23) at SKCK service points }\end{array}$ & $1.10 \%$ & $1.10 \%$ & $2.20 \%$ & $6.59 \%$ & $91.21 \%$ & $97.80 \%$ \\
\hline \multicolumn{3}{|c|}{$\begin{array}{l}\text { COMMUNITY TRUST LEVEL } \\
\text { DIRECT EVIDENCE INDICATOR }\end{array}$} & $1.09 \%$ & $1.09 \%$ & $2.19 \%$ & $7.10 \%$ & $90.71 \%$ & $97.81 \%$ \\
\hline \multirow{2}{*}{ REALIBILITY } & 3 & $\begin{array}{l}\text { Services in SKCK unit meet the } \\
\text { standard service time }\end{array}$ & $0.00 \%$ & $0.00 \%$ & $0.00 \%$ & $58.70 \%$ & $41.30 \%$ & $100.00 \%$ \\
\hline & 4 & $\begin{array}{l}\text { Speed of service permit of crowd } \\
\text { event }\end{array}$ & $0.00 \%$ & $0.00 \%$ & $0.00 \%$ & $54.55 \%$ & $45.45 \%$ & $100.00 \%$ \\
\hline \multicolumn{3}{|c|}{$\begin{array}{l}\text { COMMUNITY TRUST LEVEL } \\
\text { INDICATOR OF REALIBILITY }\end{array}$} & $0.00 \%$ & $0.00 \%$ & $0.00 \%$ & $56.62 \%$ & $43.38 \%$ & $100.00 \%$ \\
\hline $\begin{array}{l}\text { RESPONSIVEN } \\
\text { ESS }\end{array}$ & 5 & $\begin{array}{l}\text { Police discipline in providing SKCK } \\
\text { services }\end{array}$ & $0.00 \%$ & $0.00 \%$ & $0.00 \%$ & $61.96 \%$ & $38.04 \%$ & $100.00 \%$ \\
\hline
\end{tabular}


Table Cont...

\begin{tabular}{|c|c|c|c|c|c|c|c|c|}
\hline & 6 & $\begin{array}{l}\text { Police discipline in providing crowd } \\
\text { event permit services }\end{array}$ & $0.00 \%$ & $1.19 \%$ & $1.19 \%$ & $69.05 \%$ & $29.76 \%$ & $98.81 \%$ \\
\hline \multicolumn{3}{|c|}{$\begin{array}{l}\text { COMMUNITY TRUST LEVEL } \\
\text { INDICATOR OF RESPONSIVENESS }\end{array}$} & $0.00 \%$ & $0.60 \%$ & $0.60 \%$ & $65.50 \%$ & $33.90 \%$ & $99.40 \%$ \\
\hline \multirow{6}{*}{ GUARANTEE } & 7 & $\begin{array}{l}\text { The level of public comfort when } \\
\text { administering SKCK }\end{array}$ & $0.00 \%$ & $0.00 \%$ & $0.00 \%$ & $57.61 \%$ & $42.39 \%$ & $100.00 \%$ \\
\hline & 8 & $\begin{array}{l}\text { Police Capability in explaining the } \\
\text { mechanism and service requirements } \\
\text { of SKCK }\end{array}$ & $0.00 \%$ & $0.00 \%$ & $0.00 \%$ & $36.26 \%$ & $63.74 \%$ & $100.00 \%$ \\
\hline & 9 & $\begin{array}{l}\text { Ease of service procedures in the } \\
\text { SKCK service unit }\end{array}$ & $0.00 \%$ & $0.00 \%$ & $0.00 \%$ & $55.43 \%$ & $44.57 \%$ & $100.00 \%$ \\
\hline & 10 & $\begin{array}{l}\text { SKCK online service system } \\
\text { simplifies the public }\end{array}$ & $0.00 \%$ & $5.62 \%$ & $5.62 \%$ & $62.92 \%$ & $31.46 \%$ & $94.38 \%$ \\
\hline & 11 & $\begin{array}{l}\text { The level of public comfort when } \\
\text { processing crowd permits }\end{array}$ & $0.00 \%$ & $0.00 \%$ & $0.00 \%$ & $65.43 \%$ & $34.57 \%$ & $100.00 \%$ \\
\hline & 12 & $\begin{array}{l}\text { Ease of service procedures for crowd } \\
\text { permits }\end{array}$ & $0.00 \%$ & $0.00 \%$ & $0.00 \%$ & $62.20 \%$ & $37.80 \%$ & $100.00 \%$ \\
\hline \multicolumn{3}{|c|}{$\begin{array}{l}\text { COMMUNITY TRUST LEVEL } \\
\text { INDICATOR OF GUARANTEE }\end{array}$} & $0.00 \%$ & $0.94 \%$ & $0.94 \%$ & $56.64 \%$ & $42.42 \%$ & $99.06 \%$ \\
\hline \multirow{5}{*}{ COMPATIBILITY } & 13 & $\begin{array}{l}\text { Police politeness in providing SKCK } \\
\text { services }\end{array}$ & $0.00 \%$ & $0.00 \%$ & $0.00 \%$ & $49.45 \%$ & $50.55 \%$ & $100.00 \%$ \\
\hline & 14 & $\begin{array}{l}\text { SKCK services are not discriminatory } \\
\text { (people are treated fairly) }\end{array}$ & $0.00 \%$ & $1.11 \%$ & $1.11 \%$ & $4.44 \%$ & $94.44 \%$ & $98.89 \%$ \\
\hline & 15 & $\begin{array}{l}\text { The community is not charged with } \\
\text { additional fees beyond the prescribed } \\
\text { rates (Rp. 30,000) when processing } \\
\text { SKCK }\end{array}$ & $3.30 \%$ & $0.00 \%$ & $3.30 \%$ & $6.59 \%$ & $90.11 \%$ & $96.70 \%$ \\
\hline & 16 & $\begin{array}{l}\text { Police politeness in providing crowd } \\
\text { permits services }\end{array}$ & $0.00 \%$ & $0.00 \%$ & $0.00 \%$ & $55.56 \%$ & $44.44 \%$ & $100.00 \%$ \\
\hline & 17 & $\begin{array}{l}\text { Services for crowd permits are not } \\
\text { discriminatory (people are treated } \\
\text { fairly) }\end{array}$ & $1.27 \%$ & $0.00 \%$ & $1.27 \%$ & $6.33 \%$ & $92.41 \%$ & $98.73 \%$ \\
\hline \multicolumn{3}{|c|}{$\begin{array}{l}\text { COMMUNITY TRUST LEVEL } \\
\text { INDICATOR OF COMPATIBILTY }\end{array}$} & $0.91 \%$ & $0.22 \%$ & $1.13 \%$ & $24.47 \%$ & $74.39 \%$ & $98.87 \%$ \\
\hline \multicolumn{3}{|c|}{$\begin{array}{l}\text { COMMUNITY TRAST LEVEL ON SECURITY } \\
\text { INTELLIGENCE }\end{array}$} & $0.40 \%$ & $0.57 \%$ & $0.97 \%$ & $42.07 \%$ & $56.96 \%$ & $99.03 \%$ \\
\hline
\end{tabular}

Source: Processed Data

The function of Sabhara in West Sulawesi Regional Police is considered good by $95.00 \%$ of respondents. It can be interpreted by the tendency of the community to give good assessment. Respondents who stated the tendency of good assessment on each indicator are: direct evidence of $92.68 \%$, reliability of $97.64 \%$, responsiveness of $98.10 \%$, guarantee of $90.83 \%$ and compatibility of $95.75 \%$. A detailed calculation of the community's assessment of the performance of the Sabhara function in West Sulawesi Regional Police is illustrated in Table 4.

Table 4 Community Assessment of Samapta BhayangkaraFunction Performance in West Sulawesi Regional Police

\begin{tabular}{|c|c|c|c|c|c|c|c|c|}
\hline \multirow[b]{2}{*}{ INDICATOR } & \multirow[b]{2}{*}{ NO } & \multirow[b]{2}{*}{ QUESTIONS } & \multicolumn{6}{|c|}{ VERY BAD } \\
\hline & & & $\begin{array}{l}\text { VERY } \\
\text { BAD }\end{array}$ & VERY BAD & VERY BAD & VERY BAI & VERY BAD & VERY BAD \\
\hline \multirow{2}{*}{$\begin{array}{l}\text { DIRECT } \\
\text { EVIDENCE }\end{array}$} & 1 & $\begin{array}{l}\text { Comfortable feeling when meeting } \\
\text { Sabhara police }\end{array}$ & $1.89 \%$ & $8.49 \%$ & $10.38 \%$ & $47.17 \%$ & $42.45 \%$ & $89.62 \%$ \\
\hline & 2 & $\begin{array}{l}\text { Feeling safe when meeting Sabhara } \\
\text { police }\end{array}$ & $0.95 \%$ & $1.90 \%$ & $2.86 \%$ & $46.67 \%$ & $50.48 \%$ & $97.14 \%$ \\
\hline
\end{tabular}


Table Cont...

\begin{tabular}{|c|c|c|c|c|c|c|c|c|}
\hline & 3 & $\begin{array}{l}\text { The presence of the Sabhara police in } \\
\text { a crime-prone place }\end{array}$ & $0.94 \%$ & $6.60 \%$ & $7.55 \%$ & $44.34 \%$ & $48.11 \%$ & $92.45 \%$ \\
\hline & 4 & $\begin{array}{l}\text { The presence of the Sabhara police in } \\
\text { a traffic-prone area is traffic jam }\end{array}$ & $1.89 \%$ & $6.60 \%$ & $8.49 \%$ & $55.66 \%$ & $35.85 \%$ & $91.51 \%$ \\
\hline \multicolumn{3}{|c|}{$\begin{array}{l}\text { COMMUNITY TRUST LEVEL DIRECT EVIDENCE } \\
\text { INDICATORS }\end{array}$} & $1.42 \%$ & $5.90 \%$ & $7.32 \%$ & $48.46 \%$ & $44.22 \%$ & $92.68 \%$ \\
\hline \multirow{2}{*}{ RELIABILITY } & 5 & $\begin{array}{l}\text { The speed of the Sabhara police when } \\
\text { going to the crime scene }\end{array}$ & $0.00 \%$ & $2.83 \%$ & $2.83 \%$ & $51.89 \%$ & $45.28 \%$ & $97.17 \%$ \\
\hline & 6 & $\begin{array}{l}\text { The speed of Sabhara police in } \\
\text { helping victims at the crime scene }\end{array}$ & $0.00 \%$ & $1.89 \%$ & $1.89 \%$ & $51.89 \%$ & $46.23 \%$ & $98.11 \%$ \\
\hline \multicolumn{3}{|c|}{$\begin{array}{l}\text { COMMUNITY TRUST LEVEL } \\
\text { INDICATOR OF RELIABILITY }\end{array}$} & $0.00 \%$ & $2.36 \%$ & $2.36 \%$ & $51.89 \%$ & $45.75 \%$ & $97.64 \%$ \\
\hline \multirow{2}{*}{$\begin{array}{l}\text { RESPONSIVEN } \\
\text { ESS }\end{array}$} & 7 & $\begin{array}{l}\text { The level of Sabhara police } \\
\text { responsiveness in helping } \\
\text { community }\end{array}$ & $1.90 \%$ & $0.00 \%$ & $1.90 \%$ & $37.14 \%$ & $60.95 \%$ & $98.10 \%$ \\
\hline & 8 & $\begin{array}{l}\text { The readiness of Sabhara police in } \\
\text { handling crime scene }\end{array}$ & $0.00 \%$ & $1.89 \%$ & $1.89 \%$ & $52.83 \%$ & $45.28 \%$ & $98.11 \%$ \\
\hline \multicolumn{3}{|c|}{$\begin{array}{l}\text { COMMUNITY TRUST LEVEL } \\
\text { INDICATOR OF RESPONSIVENESS }\end{array}$} & $0.95 \%$ & $0.94 \%$ & $1.90 \%$ & $44.99 \%$ & $53.12 \%$ & $98.10 \%$ \\
\hline \multirow{3}{*}{ GUARANTEE } & 9 & $\begin{array}{l}\text { Sabhara police does patrol duties } \\
\text { continuously }\end{array}$ & $0.00 \%$ & $1.89 \%$ & $1.89 \%$ & $52.83 \%$ & $45.28 \%$ & $98.11 \%$ \\
\hline & 10 & $\begin{array}{l}\text { Security service of demonstrations is } \\
\text { carried out humanely }\end{array}$ & $0.95 \%$ & $16.19 \%$ & $17.14 \%$ & $32.38 \%$ & $50.48 \%$ & $82.86 \%$ \\
\hline & 11 & $\begin{array}{l}\text { Sabhara police can control the mass of } \\
\text { demonstrators so that it is not } \\
\text { anarchic (causing riots) }\end{array}$ & $4.72 \%$ & $3.77 \%$ & $8.49 \%$ & $40.57 \%$ & $50.94 \%$ & $91.51 \%$ \\
\hline \multicolumn{3}{|c|}{$\begin{array}{l}\text { COMMUNITY TRUST LEVEL } \\
\text { INDICATOR OF GUARANTEE }\end{array}$} & $1.89 \%$ & $7.28 \%$ & $9.17 \%$ & $41.93 \%$ & $48.90 \%$ & $90.83 \%$ \\
\hline \multirow{4}{*}{ COMPATIBILITY } & 12 & $\begin{array}{l}\text { Sabhara Police does not carry out } \\
\text { illegal levies in patrol duties }\end{array}$ & $2.83 \%$ & $4.72 \%$ & $7.55 \%$ & $10.38 \%$ & $82.08 \%$ & $92.45 \%$ \\
\hline & 13 & $\begin{array}{l}\text { Sabhara Police is humanism (not } \\
\text { arrogant) }\end{array}$ & $0.00 \%$ & $3.81 \%$ & $3.81 \%$ & $20.00 \%$ & $76.19 \%$ & $96.19 \%$ \\
\hline & 14 & $\begin{array}{l}\text { Sabhara police do not ask for } \\
\text { compensation in conducting security } \\
\text { activities (escort and guarding) }\end{array}$ & $1.89 \%$ & $2.83 \%$ & $4.72 \%$ & $10.38 \%$ & $84.91 \%$ & $95.28 \%$ \\
\hline & 15 & $\begin{array}{l}\text { Police Sabhara does not act of } \\
\text { violence in demonstration security } \\
\text { services }\end{array}$ & $0.00 \%$ & $0.94 \%$ & $0.94 \%$ & $16.98 \%$ & $82.08 \%$ & $99.06 \%$ \\
\hline \multicolumn{3}{|c|}{$\begin{array}{l}\text { COMMUNITY TRUST LEVEL INDICATORS } \\
\text { INDICATOR OF COMPATIBILITY }\end{array}$} & $1.18 \%$ & $3.08 \%$ & $4.25 \%$ & $14.43 \%$ & $81.31 \%$ & $95.75 \%$ \\
\hline \multicolumn{3}{|c|}{$\begin{array}{l}\text { COMMUNITY TRUST LEVEL } \\
\text { ON SABHARA FUNCTION }\end{array}$} & $1.09 \%$ & $3.91 \%$ & $5.00 \%$ & $40.34 \%$ & $54.66 \%$ & $95.00 \%$ \\
\hline
\end{tabular}

Source: Processed Data

The performance of the Binmas function in the West Sulawesi Regional Police was assessed with a tendency towards good assessment by $99.27 \%$ of respondents. Indicators of direct evidence, reliability, responsiveness, guarantee and compatibility acquired good assessment by respondents in a row: $98.59 \% ; 98.30 \% ; 100.00 \% ; 100.00 \% ; 99.44 \%$. In detail the results of the assessment are illustrated in Table 5. 
Table 5

Community Assessment of Community Development FunctionPerformance In West Sulawesi Regional Police

\begin{tabular}{|c|c|c|c|c|c|c|c|c|}
\hline \multirow[b]{2}{*}{ INDICATOR } & \multirow[b]{2}{*}{ NO } & \multirow[b]{2}{*}{ QUESTIONS } & \multicolumn{6}{|c|}{ VERY BAD } \\
\hline & & & $\begin{array}{l}\text { VERY } \\
\text { BAD }\end{array}$ & VERY BAD & VERY BAD & VERY BAD & VERY BAD & VERY BAD \\
\hline \multirow{4}{*}{$\begin{array}{l}\text { DIRECT } \\
\text { EVIDENCE }\end{array}$} & 1 & $\begin{array}{l}\text { Visiting activity to the community by } \\
\text { Bhabinkamtibmas }\end{array}$ & $0.00 \%$ & $2.27 \%$ & $2.27 \%$ & $39.77 \%$ & $57.95 \%$ & $97.73 \%$ \\
\hline & 2 & $\begin{array}{l}\text { The existence of Bhabinkamtibmas in } \\
\text { the community environment }\end{array}$ & $0.00 \%$ & $0.00 \%$ & $0.00 \%$ & $29.21 \%$ & $70.79 \%$ & $100.00 \%$ \\
\hline & 3 & $\begin{array}{l}\text { Bhabinkamtibmas has provided } \\
\text { information of community security and } \\
\text { order to the public }\end{array}$ & $0.00 \%$ & $3.37 \%$ & $3.37 \%$ & $30.34 \%$ & $66.29 \%$ & $96.63 \%$ \\
\hline & 4 & $\begin{array}{l}\text { Bhabinkamtibmas are directly involved } \\
\text { in social activities (attending funerals, } \\
\text { religious activities, handling natural } \\
\text { disasters etc.) }\end{array}$ & $0.00 \%$ & $0.00 \%$ & $0.00 \%$ & $34.83 \%$ & $65.17 \%$ & $100.00 \%$ \\
\hline \multicolumn{3}{|c|}{$\begin{array}{l}\text { COMMUNITY TRYST LEVEL } \\
\text { DIRECT EVIDENCE }\end{array}$} & $0.00 \%$ & $1.41 \%$ & $1.41 \%$ & $33.54 \%$ & $65.05 \%$ & $98.59 \%$ \\
\hline \multirow{2}{*}{ REALIBILITY } & 5 & $\begin{array}{l}\text { Members of Bhabinkamtibmas have } \\
\text { data on community members in the } \\
\text { area of duty }\end{array}$ & $1.12 \%$ & $1.12 \%$ & $2.25 \%$ & $50.56 \%$ & $47.19 \%$ & $97.75 \%$ \\
\hline & 6 & $\begin{array}{l}\text { Data of disturbances security and order } \\
\text { in the area of duty }\end{array}$ & $0.00 \%$ & $1.15 \%$ & $1.15 \%$ & $25.29 \%$ & $73.56 \%$ & $98.85 \%$ \\
\hline \multicolumn{3}{|c|}{$\begin{array}{l}\text { COMMUNITY TRUST LEVEL } \\
\text { INDICATOR OF REALIBILITY }\end{array}$} & $0.56 \%$ & $1.14 \%$ & $1.70 \%$ & $37.92 \%$ & $60.38 \%$ & $98.30 \%$ \\
\hline $\begin{array}{l}\text { RESPONSIVENE } \\
\text { SS }\end{array}$ & 7 & $\begin{array}{l}\text { Attitudeof Bhabinkamtibmas members } \\
\text { when the community asks for help }\end{array}$ & $0.00 \%$ & $0.00 \%$ & $0.00 \%$ & $25.56 \%$ & $74.44 \%$ & $100.00 \%$ \\
\hline \multicolumn{3}{|c|}{$\begin{array}{l}\text { COMMUNITY TRUST LEVEL } \\
\text { INDICATOR OF RESPONSIVENESS }\end{array}$} & $0.00 \%$ & $0.00 \%$ & $0.00 \%$ & $25.56 \%$ & $74.44 \%$ & $100.00 \%$ \\
\hline \multirow{5}{*}{ GUARANTEE } & 8 & $\begin{array}{l}\text { The usefulness of visiting activities to } \\
\text { the community by Bhabinkamtibmas }\end{array}$ & $0.00 \%$ & $0.00 \%$ & $0.00 \%$ & $23.33 \%$ & $76.67 \%$ & $100.00 \%$ \\
\hline & 9 & $\begin{array}{l}\text { Visiting to the community by police is a } \\
\text { media to build relationships to be more } \\
\text { harmonious between the community } \\
\text { and the police }\end{array}$ & $0.00 \%$ & $0.00 \%$ & $0.00 \%$ & $22.22 \%$ & $77.78 \%$ & $100.00 \%$ \\
\hline & 10 & $\begin{array}{l}\text { Bhabinkamtibmas presence provides } \\
\text { security in the community }\end{array}$ & $0.00 \%$ & $0.00 \%$ & $0.00 \%$ & $24.44 \%$ & $75.56 \%$ & $100.00 \%$ \\
\hline & 11 & $\begin{array}{l}\text { The role of Bhabinkamtibmas increases } \\
\text { public awareness about the importance } \\
\text { of security, public order (Kamtibmas) }\end{array}$ & $0.00 \%$ & $0.00 \%$ & $0.00 \%$ & $18.89 \%$ & $81.11 \%$ & $100.00 \%$ \\
\hline & 12 & $\begin{array}{l}\text { The role of Bhabinkamtibmas in the } \\
\text { community makes the environment } \\
\text { safeguard (Kamling) work well }\end{array}$ & $0.00 \%$ & $0.00 \%$ & $0.00 \%$ & $35.56 \%$ & $64.44 \%$ & $100.00 \%$ \\
\hline \multicolumn{3}{|c|}{$\begin{array}{l}\text { COMMUNITY TRUST LEVEL } \\
\text { INDICATOR OF GUARANTEE }\end{array}$} & $0.00 \%$ & $0.00 \%$ & $0.00 \%$ & $24.89 \%$ & $75.11 \%$ & $100.00 \%$ \\
\hline \multirow[t]{2}{*}{ COMPATIBILITY } & 13 & $\begin{array}{l}\text { Ability of Bhabinkamtibmas in } \\
\text { resolving conflicts / disputes among } \\
\text { community }\end{array}$ & $0.00 \%$ & $0.00 \%$ & $0.00 \%$ & $38.89 \%$ & $61.11 \%$ & $100.00 \%$ \\
\hline & 14 & $\begin{array}{l}\text { Bhabinkamtibmas do not ask for fees in } \\
\text { community service activities }\end{array}$ & $0.00 \%$ & $1.12 \%$ & $1.12 \%$ & $3.37 \%$ & $95.51 \%$ & $98.88 \%$ \\
\hline \multicolumn{3}{|c|}{$\begin{array}{l}\text { COMMUNITY TRUST LEVEL } \\
\text { INDICATOR OF COMPATIBILITY }\end{array}$} & $0.00 \%$ & $0.56 \%$ & $0.56 \%$ & $21.13 \%$ & $78.31 \%$ & $99.44 \%$ \\
\hline \multicolumn{3}{|c|}{$\begin{array}{l}\text { COMMUNITY TRUST LEVEL ON COMMUNITY } \\
\text { DEVELOPMENT FUNCTIONS }\end{array}$} & $0.11 \%$ & $0.62 \%$ & $0.73 \%$ & $28.61 \%$ & $70.66 \%$ & $99.27 \%$ \\
\hline
\end{tabular}

Source: Processed Data 
Based on the results illustrated data in Table 1 to 5 , it can be seen that the community development functions gets the best performance assessment among the four defined functions. Schematically illustrated in Table 6.

Table 6

Trends in Good Community Assessment Against the Quality of Police Services in 2018 in Five Functions and Five Aspects of Police Services in West Sulawesi Regional Police

\begin{tabular}{|c|c|c|c|c|c|c|}
\hline \multirow{2}{*}{ FUNCTIONS } & \multicolumn{5}{|c|}{ INDICATOR } & \multirow{2}{*}{$\begin{array}{c}\text { RATE OF } \\
\text { FUNCTIONS }\end{array}$} \\
\hline & DIRECT EVIDENCE & REALIBILITY & RESPONSIVENESS & GUARANTEE & COMPATIBILITY & \\
\hline TRAFFIC & $87.14 \%$ & $92.79 \%$ & $96.52 \%$ & $95.19 \%$ & $95.55 \%$ & $93.44 \%$ \\
\hline RESERSE AND CRIME & $99.08 \%$ & $93.64 \%$ & $97.30 \%$ & $96.69 \%$ & $91.71 \%$ & $95.68 \%$ \\
\hline INTELLEGENCE & $97.81 \%$ & $100.00 \%$ & $99.40 \%$ & $99.06 \%$ & $98.87 \%$ & $99.03 \%$ \\
\hline SABHARA & $92.68 \%$ & $97.64 \%$ & $98.10 \%$ & $90.83 \%$ & $95.75 \%$ & $95.00 \%$ \\
\hline $\begin{array}{l}\text { COMMUNITY } \\
\text { DEVELOPMENT } \\
\text { FUNCTION }\end{array}$ & $98.59 \%$ & $98.30 \%$ & $100.00 \%$ & $100.00 \%$ & $99.44 \%$ & $99.27 \%$ \\
\hline INDICATOR RATE & $95.06 \%$ & $96.47 \%$ & $98.27 \%$ & $96.35 \%$ & $96.26 \%$ & $96,48 \%$ \\
\hline
\end{tabular}

Source: Processed Data

The second discussion of the research is regarding management of Police Performance in West Sulawesi Regional Police. The management of Polri's performance management in Poda West Sulawesi was conducted with three programs, namely: Mi'oro Siola (sitting together), the synergy of the four pillars and Shubuh safari which was carried out togetherby the Regional Police and Regional Government. The Mi'oro Siola program was launched by the Head of West Sulawesi Regional Police (Kapolda) at Marmatunnajah Mosque, Musa Karim Street, Karema Village, Mamuju District, West Sulawesi on November 4, 2016.

The synergy of the four pillars implemented in West Sulawesi Regional Police aimed to realize Community Security and Order (Kamtibmas) and became a place for village officials to consult about the utilization of village funds based on target. Four pillars consist of Bhabinkamtibmas, Village Heads, Babinsa and Religious Extension (Da'I Kamtibmas), one of which aims to optimize the acceleration of village development in jurisdiction of the West Sulawesi Regional Police. This optimization was realized through fostering and supervising the use of village funds carried out by four pillars.

The third program relating to the management of the performance of the National Police in West Sulawesi Regional Police is Shubuh safari (Shubuh prayer with different mosque places), twice a week, hold on Tuesday and Saturday. The program was carried out in harmony between Mamuju Regional Government and West Sulawesi Regional Police. This program aims to bring the police closer to the community of West Sulawesi. In addition to the Shubuh safari program, at the same time i'tikaf and tauziah were also conducted.

The implementation of three programs and the achievement of determined performance targets depend on the accuracy of the performance management. Management can be developed with a pattern of partnership, empowerment and integration through health work environment. The health work environment has a positive impact on the work culture so that it is easier to build and realize a positive work culture. The partnership pattern aims to instill understanding and acceptance of work directions and targets for Indonesian National Police personnel. Empowerment will have positive 
implications for the competence, role and authority of Indonesian Police personnel, so that the integration of performance management can produce a positive assessment of the National Police performance.

\section{Conclusions}

Public perceptions on performance of the National Police in the West Sulawesi Regional Police are positive, indicated by informants who stated that the performance of National Police personnel in West Sulawesi Regional Police tends to be as good as $96.48 \%$. It can be interpreted that the community considered the presence and serviceof police in five operational functions to be good. In addition, the community also considered that police services had been provided on time and had accurate results. The community also considered that West Sulawesi Regional Police personnel had adequate knowledge and skills, so that the community could trust the police. Police personnel in public view have a willingness to help and have a sense of empathy. Police services in West Sulawesi Regional Police are also considered to have good quality because they did their dutyaccordance with the procedures, compliance and provisions of the cost determined.

The management of national police performance management in West Sulawesi Regional Police was conducted with three excellent programs, namely: Mi'oro Siola (sitting together), synergy of four pillars and Safari Shubuh. The three programs aim to bring the National Police closer to the community, so that the National Police will more easily identify the needs, desires and expectations of the community, therefore the implementation of police duties in protecting, protecting and serving the community, holding public security and order and enforcing the law can be achieved optimally. The pattern of partnership, empowerment and integration through the health of the work environment are needed to build the sustainableprograms and maintaining the positive performance of National Police personnel in West Sulawesi Regional Police.

\section{References}

Colquitt, Jason A., Lepine J.A\& Wesson M.J (2013).Organizational behaviour, improving performnce and commitment in the workplace (pp. 3014). New York, NY: McGraw-Hill Companies

Greenberg, J \&Baron, R.A (2008). Behavior in Organizations(pp. 159), New Jersey: Prentice Hall

Ivancevich, J.M., Konopaske,R \&Matteson, M.T (2011). Organizational behavior and management (pp. 74) New York: McGraw-Hill Irwin

Mayastinasari, V (2018). Tingkat kepercayaan masyarakat terhadap kinerja Polri (pp. 1). Jakarta: Pusat Penelitian dan Pengembangan POLRI

Schermerhorn, J.R., Hunt, J,G \&Osborn, R.N (2008).Organizational behavior (pp. 68). Jefferson City: John Wiley \& Sons, Inc.

Solikin (2011). Pelayanan prima (pp. 2). Jakarta: Inti Prima Promosindo

Tjiptono,Fandy (1997). Prinsip-prinsip TQS(pp. 119). Yogyakarta: Andi 